\title{
Literaturverzeichnis.
}

Benecke, System des Assekuranz- und Bodmereiwesens nsw. (Benecke) Hamburg $1805-1810$.

Bruck, in Die Reichsgerichtspraxis im deutschen Rechtsleben. Festgabe der juristischen Fakultäten zum 50jährigen Bestehen des Reichsgerichts. Bd. 4, Berlin, Leipaig 1929. (Bruck, Festgabe).

- Reichsgesetz über den Versicherungsvertrag nebst dem zugehörigen Einführungsgesetz vom 30. Mai 1908, 6. Aufl. 1929. (Bruck.)

Ehrenberg, Das Interesse im Versicherungsrecht. München-Leipzig 1915. (Ehrenberg, Interesse.)

- Versicherungsrecht I. Leipzig 1893. (Ehrenberg, Versicherungsrecht.)

Elkan, Die Bedeutung des Interesses für die Veräußerung der versicherten Sache. Hamburg 1928. (Flkan).

Gerhard-Hagen, Kommentar zum deutschen Reichsgesetz über den Versicherungavertrag. Berlin 1908.

Ha gen, in Ehrenbergs Handbuch, Bd. 8, 1. Abtlg.

Helmer, Die Geschichte der privaten Fenerversicherung in den Herzogtümern Schleswig und Holstein. Berlin 1925. (Helmer.)

Josef, Reichsgesetz über den Versicherungovertrag. Berlin 1908.

Kisch, Handbuch des Privatversicherungsrechts. München 1922. (Kisch.)

I.enné, Das Vervicherangsgeschăft für fremde Rechnung. Marburg 1911. (Lenne.)

Ritter, Das Reeht der Seeversicherang, ein Kommentar zu den Allgemeinen Deutschen Seeversicherungsbedingungen. Hamburg 1922. (Ritter.)

Schneider, Reichsgesetz ther den Versicherungsvertrag. Berlin 1908.

Sprinz, Reichsgesetz über den Versicherungevertrag. Berlin 1926.

We ygand, Die Grundzilge der Kundenversicherung, (Generalversicherung der Speditionsund Lagergiuter) mit einer Ënleitung über das Interesse als Element der Sachversicherung. Berlin 1914. (Weygand.)

\section{Materialien und Zeitschriften.}

Allgemeine Deutsche Seeversicherungsbedingungen von 1919. (ADS.)

Allgemeines Landrecht für die Preußischen Staaten. (PrALR.)

Der Stadt Hamburg Assecuranz und Haverey-Ordnung von 1731. Hamburg 1807.

Burgerliches Gesetzbuch. (BGB.)

Handelagesetzbuch. (HGB.)

HansGZ. = Hanseatische Gerichtszeitung.

HansRZ. = Hanseatiache Rechtszeitschrift.

HansRGZ. = Hanseatische Rechts- und Gerichtszeitschrift.

Jherings Jahrbulcher für die Dogmatik des bürgerlichen Rechts. (Jb. Jb.)

Juristische Rundschan für die Privatversicherung. (JR.)

Juristische Wochenschrift. (JW.)

Leipziger Zeitschrift für deutsches Recht. (LZ.)

Materialien zu den ADS., herausgegeben von Bruck, Hamburg 1919.

Protokolle zum ADHGB. Würzburg 1858.

Entscheidungen des Reichsgerichts in Zivilsachen. (RG.)

Entscheidungen des Reichsoberhandelsgerichts. (ROHG.)

Seufferts Archiv für Entscheidungen der Obersten Gerichte in den Deutschen Staaten. (Seuff. Arch.)

Urteilssammlung des Hamburgischen Seminars für Versicherungswissenschaft.

Veröffentlichungen des Reichsaufsichtsamts für Privatversicherang. (VA.)

Reichsgesetz über den Versicherungsvertrag vom 30. Mai 1908. (VVG.)

Zeitschrift für die gesamte Versicherungswissenschaft. (Z. f. ges. V. W.)

Zeitachrift für Versicherungswesen. (Z.f. V. Wes.) 